\title{
Hypoxia-inducible factor 1- $\alpha$ promotes colon cell proliferation and migration by upregulating AMPK-related protein kinase 5 under hypoxic conditions
}

\author{
JI KUI PENG ${ }^{1}$, SHI QIANG SHEN ${ }^{1}, \mathrm{JU} \mathrm{WANG}^{2}, \mathrm{HONG}^{\mathrm{WEI}} \mathrm{JIANG}^{2}$ and YONG QIANG WANG ${ }^{2}$ \\ ${ }^{1}$ Department of Endoscopic Surgery, Renmin Hospital of Wuhan University, Wuhan, Hubei 430060; \\ ${ }^{2}$ Department of Gastrointestinal Surgery, Inner Mongolia People's Hospital, Hohhot 010000, Inner Mongolia, P.R. China
}

Received October 13, 2016; Accepted September 13, 2017

DOI: $10.3892 / \mathrm{ol} .2018 .7748$

\begin{abstract}
Hypoxia is a common characteristic of solid tumors. Previous studies have reported that the tumor invasion-associated factor, AMPK-related protein kinase 5 (ARK5), is associated with a poor prognosis in colon cancer. However, whether or not ARK5 is involved in hypoxia is unclear. The aim of present study was to investigate the association between the expression of ARK5 and that of hypoxia-inducible factor 1- $\alpha$ (HIF1- $\alpha)$. Samples from 60 patients with colon cancer were collected and immunohistochemistry was used to detect the expression of ARK5 and HIF1- $\alpha$ within them. Western blot analysis and reverse transcription polymerase chain reaction were used to detect the expression of ARK5 in an SW480 cell line under hypoxic conditions. Cell Counting kit-8 and Transwell assays were used to study the function of ARK5 under hypoxic conditions. According to the immunohistochemistry results, ARK5 and HIF1- $\alpha$ staining was significantly associated with Tumor-Node-Metastasis stage, tumor grade, lymph node metastasis and liver metastasis. Spearman's correlation analysis revealed a correlation between the expression of ARK5 and that of HIF1- $\alpha$. This finding was also verified under hypoxic conditions in the SW480 cell line, in which the expression of ARK5 increased over time. Further cellular function experiments revealed that suppression of ARK5 inhibited cell viability and migration under hypoxic conditions. The present study has suggested that ARK5 expression in colon cancer cells is upregulated by HIF1- $\alpha$ under hypoxic conditions and that ARK5 serves an important role in cell proliferation and migration under hypoxic stress.
\end{abstract}

Correspondence to: Dr Shi Qiang Shen, Department of Endoscopic Surgery, Renmin Hospital of Wuhan University, 238 Jiefang Road, Wuchang, Wuhan, Hubei 430060, P.R. China

E-mail: swsw2218@126.com

Key words: AMPK-related protein kinase 5, hypoxia-inducible factor 1- $\alpha$, colon cancer, hypoxia, proliferation, migration

\section{Introduction}

Colon cancer is one of the most lethal solid tumors of the gastrointestinal tract, and is the third most common cancer in men and the second in women (1). It was estimated that 134,490 new cases and 49,190 mortalities would occur in 2016 (2). Surgical resection is the most common treatment for all stages of colon cancer, whereas for intermediate and advanced colon cancer, chemotherapy and radiation therapy may be additional methods used to protect against tumor progression (3). Despite the substantial progress that has been made in the treatment of colon cancer, patients continue to develop advanced cancer, resulting in a poor prognosis. Therefore, understanding the mechanisms underlying colon cancer is essential for an effective strategy to improve the present situation.

AMPK-related protein kinase 5 (ARK5), the fifth member of the $5^{\prime}$ adenosine monophosphate-activated protein kinase catalytic subunit family, is a tumor invasion-associated factor regulated by Akt signaling (4). Suzuki et al $(5,6)$ demonstrated that ARK5 induces tumor cell survival during nutrient starvation by inhibiting caspase- 8 activation and that it resists the Fas ligand/Fas system by negatively regulating procaspase-6 phosphorylation at $\mathrm{Ser}^{257}$. In further studies, it was observed that ARK5 was involved in hypoxia-induced tumor cell tolerance to glucose starvation regulated by the transforming growth factor- $\beta$ signaling pathway (7), and that ARK5 was downstream of NDR2 during activation of insulin-like growth factor-1 signaling (8). Previous studies reported that ARK5 was overexpressed in numerous malignancies, including breast cancer, colorectal carcinoma, hepatocellular carcinoma and gliomas, and that patients overexpressing ARK5 often had a poor prognosis (9-12). ARK5 was also a direct target of large musculoaponeurotic fibrosarcoma proteins and this activation was mediated through MAF-recognition element sequences (13). However, the relationship between ARK5 and HIFla remains unclear.

The transcription factor, hypoxia-inducible factor 1 (HIF1), has been reported to be one of the key prognostic tumor factors that is accumulated and detectable under hypoxic conditions (14). HIF1 is a heterodimer consisting of two components; HIF1- $\alpha$ and HIF1- $\beta$. HIF1- $\alpha$ is oxygen-regulated and determines the activity of HIF-1 (15). As an important 
regulator in hypoxic conditions, HIF-1 $\alpha$ regulates tumor metabolism, proliferation, apoptosis, metastasis, inflammation and angiogenesis (16). In a study by Wang et al (17), HIF1- $\alpha$ was overexpressed in colon cancer and was associated with a poor prognosis.

In the present study, the relationship between ARK5 and HIF1a in colon cancer was investigated in order to identify novel molecular targets for the treatment of colon cancer.

\section{Materials and methods}

Patients and samples. Tissue samples were obtained from 60 patients with colon cancer ( 32 men and 28 women; aged 30-80 years) who had undergone surgery at the Department of Endoscopic Surgery, Renmin Hospital of Wuhan University (Hubei, China). All patients had received an intraoperative pathological diagnosis and none of the patients had previously undergone radiotherapy or chemotherapy. Patient charts were reviewed to obtain clinical data regarding age, sex, Tumor-Node-Metastasis (TNM) staging (18), tumor histologic grade (18) and lymph node metastasis. Written informed consent was obtained from all patients, and all experiments were approved by the medical ethics committee of Renmin Hospital of Wuhan University (Wuhan, China).

Cell lines and cell culture. An SW480 cell line was purchased from the Shanghai Cell Bank at the Chinese Academy of Sciences (Shanghai, China). This cell line was cultured in RPMI-1640 medium (Gibco; Thermo Fisher Scientific, Inc. Waltham, MA, USA) supplemented with $10 \%$ fetal bovine serum (FBS; Hyclone; GE Healthcare Life Sciences, Logan, UT, USA) at $37^{\circ} \mathrm{C}$ in a humidified incubator containing $5 \%$ $\mathrm{CO}_{2}$. A 3-chamber air incubator flushed with a gas mixture of $5 \% \mathrm{CO}_{2}$ and $94 \% \mathrm{~N}_{2}$ at $37^{\circ} \mathrm{C}$ was used to create the hypoxic conditions. The final $\mathrm{O}_{2}$ pressure of the medium was measured at $1 \%$.

Total RNA extraction and reverse transcription quantitative polymerase chain reaction (RT-qPCR). A TRIzol Total RNA Extraction kit (Invitrogen; Thermo Fisher Scientific, Inc.) was used for total RNA extraction from the SW480 cell line; cDNA was synthesized using a ReverTra Ace- $\alpha$ reverse transcription kit (Invitrogen; Thermo Fisher Scientific, Inc.). For RT-qPCR analysis, the Roche LightCycler (Roche Applied Science, Penzberg, Germany) was used with the Takara SYBR Premix Extaq system (Takara Biotechnology Co., Ltd., Dalian, China). All procedures were performed according to the manufacturer's protocols. The thermocycling conditions were as follows: Cycle 1 at $95^{\circ} \mathrm{C}$ for $30 \mathrm{sec}$, cycle 2,40 cycles at $95^{\circ} \mathrm{C}$ for $5 \mathrm{sec}$ and at $60^{\circ} \mathrm{C}$ for $30-60 \mathrm{sec}$, followed by dissociation. Primers were synthesized by Shanghai Sangon Biological Engineering Technology Services Co., Ltd. (Shanghai, China). The nucleotide sequences of the primers were as follows: $\beta$-actin forward, 5'-ATGGGGAAGGTGAAGGTCG-3' and reverse, 5'-ATG GGGAAGGTGAAGGTCG-3'; ARK5 forward, 5'-GGGAAG GTGAAGGTCG-3' and reverse, 5'-GGGAAGGTGAAGGTC G-3'; and HIF1- $\alpha$ forward, 5'-ATCCATGTGACCATGAGG AAATG-3' and reverse, 5'-ATCCATGTGACCATGAGGAAA TG-3'. Each sample was taken in triplicate, $\beta$-actin was used as an internal reference and the $2^{-\Delta \Delta C q}$ method (19) was used to analyze the PCR results.

Western blot analysis. Total protein was extracted from harvested SW480 cells using a protein extraction kit (Beyotime Institute of Biotechnology, Haimen, China). Total protein was quantified according to the protocol of the Pierce BCA-200 Protein Assay kit (Thermo Scientific ${ }^{\mathrm{TM}}$, Waltham, USA) and denatured by boiling (ARK5=74 kD, HIF1- $\alpha=120 \mathrm{kD}$, $\beta$-actin $=43 \mathrm{kD}$ ), separated by $10 \%$ SDS-PAGE Precast Gel and transferred onto polyvinylidene fluoride membranes. The membranes were then blocked by $5 \%$ milk (at $37^{\circ} \mathrm{C}$ for $1 \mathrm{~h}$ ) prior to being incubated with primary antibodies overnight at $4^{\circ} \mathrm{C}$. The membranes were then washed 3 times with TBST (Tris-buffered saline with $0.1 \%$ Tween-20), prior to being incubated with a secondary horseradish peroxidase goat anti-rabbit/anti-mouse immunoglobulin $\mathrm{G}(\mathrm{H}+\mathrm{L})$ antibody (dilution 1:2,000; cat no. ab97051/ ab97023; Abcam, Cambridge, UK) for $2 \mathrm{~h}$ at $37^{\circ} \mathrm{C}$. Finally, the membranes were visualized using a chemiluminescence kit (Beyotime Institute of Biotechnology) on a Bio-Rad imaging system (Bio-Rad Laboratories, Inc., Hercules, CA, USA). The primary antibodies used in the present study were as follows: HIF1- $\alpha$ (cat no. ab114977), ARK5 (cat no. ab37641) and $\beta$-actin (cat no. ab8227; all at a dilution of 1:1,000; Abcam).

Immunohistochemical analysis. Tissues of 60 patients with colon cancer from our hospital were sent to Shanghai OUTDO Biotech Co., Ltd and converted into a microarray, and immunostaining was performed as per the following protocol. Tissue microarrays (thickness of $4 \mu \mathrm{m}$ ) were deparaffinized and placed in a solution of absolute methanol and $0.3 \%$ hydrogen peroxide for $30 \mathrm{~min}$. Antigen retrieval was then performed by heating the slides in citrate buffer ( $\mathrm{pH}$ 6.2) in a microwave oven at $95^{\circ} \mathrm{C}$ for $20 \mathrm{~min}$ and washing them in PBS 3 times prior to staining with immunoperoxidase. Slides were incubated in a humidified chamber with blocking agent (5\% FBS) for $30 \mathrm{~min}$ at room temperature. The slides were then incubated overnight with anti-ARK5 antibody (dilution, 1:100; cat no. ab37641; Abcam) and anti-HIF1- $\alpha$ antibody (dilution, 1:100; cat no. ab114977; Abcam) in 5\% FBS at $4^{\circ} \mathrm{C}$. Following being washed with phosphate-buffered saline, the slides were incubated with horseradish peroxidase-conjugated goat anti-rabbit immunoglobulin for $40 \mathrm{~min}$ at room temperature. Results were visualized by reaction with diaminobenzidine and counterstained with hematoxylin (at room temperature for $3 \mathrm{~min}$ ). For each sample, images of 5 randomly-selected areas were visualized using a light microscope (magnification, x100) and captured using a high-power objective lens camera (Axiocam ERc 5s; Zeiss GmbH, Jena, Germany) under the same conditions. Evaluation of immunohistochemistry results was performed by 2 independent observers to determine the percentage of positive cells following inspection of all fields in the sections, as described previously (20).

Transfection of the SW480 cell line with small interfering (si)RNA. Human-specific HIF1- $\alpha$ and ARK5 siRNA were designed, constructed and purified by Jima Biotech Co. (Shanghai, China). Their respective sequences were as follows: HIF1- $\alpha$, 5'-GGAAATGAGAGAAATGCTTAC-3'; and ARK5, 

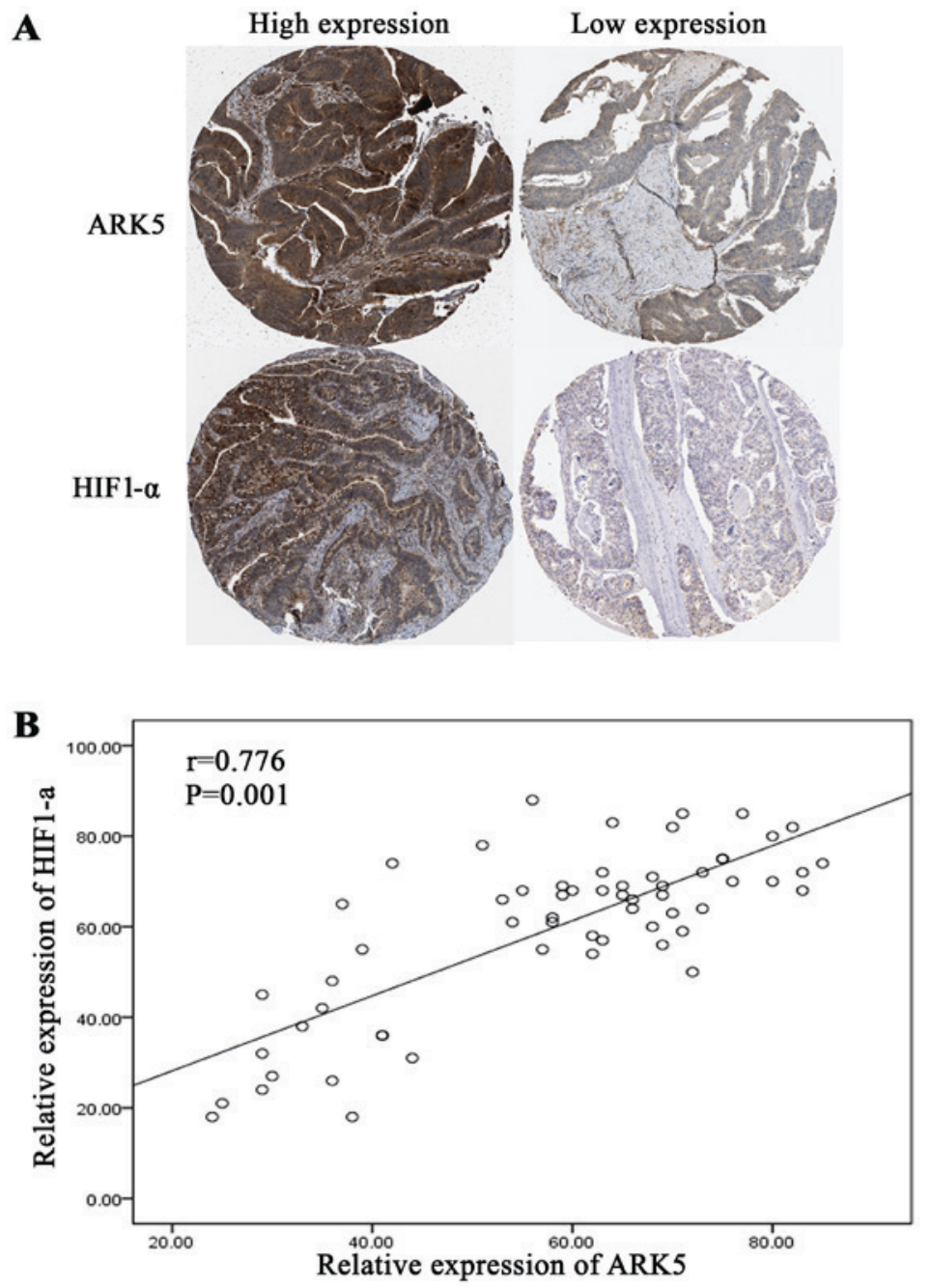

Figure 1. (A) Immunohistochemical staining of HIF1- $\alpha$ and ARK5 in different colon cancer tissues. Cells with high expression are stained brown, while cells with low expression lack staining. (B) Spearman's correlation analysis was performed on the expression of ARK5 and HIF1- $\alpha$. $r=0.776$, P=0.001. HIF1- $\alpha$, hypoxia-inducible factor 1- $\alpha$; ARK5, AMPK-related protein kinase 5.

5'-GAAGTTATGCTTTATTCAC-3'. After determining the best effect of interference and the best transfer multiplicity of infection (60) through the fluorescence camera, and performing RT-qPCR and western blot analysis, SW480 cells were seeded onto 6-well plates at a concentration of $0.3 \times 10^{5}$ per well (20-30\% confluence) 1 day prior to siRNA transfection. The $5 \mu \mathrm{l}$ of $50 \mathrm{nM}$ HIF1- $\alpha$ siRNA, ARK5 siRNA and negative control were transfected into different cells with Lipofectamine 2000 reagent (Invitrogen; Thermo Fisher Scientific, Inc.). Following incubation at room temperature for $6 \mathrm{~h}$, medium was replaced with fresh RPMI-1640. According to the indicated times, the cells were harvested for subsequent studies.

Cell counting kit (CCK)-8 and Transwell assay. ARK5 siRNA-transfected cells were collected and then seeded at a density of $1.0 \times 10^{4}$ cells/well onto 96 -well plates. Cell viability was subsequently measured using CCK-8 (Dojindo Molecular Technologies, Inc., Kumamoto, Japan) at 8, 24, 48 and $72 \mathrm{~h}$. Other procedures were according to the manufacturer's protocols. Next, $1 \times 10^{5}$ cells transfected with ARK5 siRNA were seeded into a serum-free minimum essential medium (MEM) in the upper chamber on an insert coated without Matrigel (cat. no. PSET010R5; EMD Millipore, Billerica, MA, USA). In the lower chamber, medium was swapped for MEM with $10 \%$ FBS. The cells were incubated for $24 \mathrm{~h}$. Following fixation in room temperature with $100 \%$ methanol for $10 \mathrm{~min}$ and staining by crystal violet for $5 \mathrm{~min}$ at room temperature, cells on the bottom surface that had invaded across the membranes were counted and images were captured under a light microscope (magnification, x100) using a camera (Axiocam ERc 5s; Zeiss $\mathrm{GmbH}$ ). All experiments were performed in triplicate.

Cell apoptosis. SW480 cells transfected with ARK5 siRNA or control were trypsinized and washed 3 times with pre-chilled PBS buffer and resuspended in $100 \mu \mathrm{l}$ PBS at $1 \times 10^{6}$ cells $/ \mathrm{ml}$ after $24 \mathrm{~h}$ of treatment in hypoxic conditions. Apoptotic SW480 cells were measured by staining with fluorescein isothiocyanate and a propidium iodide (PI) kit (Annexin V-FITC/PI Apoptosis Assay kit, 70-AP101-100; MultiSciences Biotech Co. Ltd., Hangzhou, China) according to the manufacturer's protocol (at room temperature for $30 \mathrm{~min}$ in the dark), and were analyzed using a flow cytometer (BD FACSCalibur; BD Biosciences) and FlowJo software 7.6 (FlowJo LLC, Ashland, OR, USA). 
Table I. Association between HIF1- $\alpha$ and ARK5 expression and clinicopathological features of colon cancer.

\begin{tabular}{|c|c|c|c|c|c|c|}
\hline \multirow[b]{2}{*}{ Variables } & \multicolumn{3}{|c|}{ HIF1- $\alpha$ expression } & \multicolumn{3}{|c|}{ ARK5 expression } \\
\hline & High & Low & P-value & High & Low & P-value \\
\hline \multicolumn{7}{|l|}{ Age, years } \\
\hline$\leq 60$ & 26 & 10 & 0.304 & 24 & 12 & 0.282 \\
\hline$>60$ & 20 & 4 & & 19 & 5 & \\
\hline \multicolumn{7}{|l|}{ Sex } \\
\hline Male & 28 & 4 & 0.143 & 25 & 8 & 0.263 \\
\hline Female & 18 & 10 & & 18 & 9 & \\
\hline \multicolumn{7}{|l|}{ TNM staging } \\
\hline I-II & 12 & 14 & $0.001^{\mathrm{a}}$ & 11 & 15 & $0.008^{\mathrm{a}}$ \\
\hline III-IV & 34 & 0 & & 32 & 2 & \\
\hline \multicolumn{7}{|l|}{ Grade } \\
\hline Well/moderate & 10 & 12 & $0.005^{\mathrm{a}}$ & 8 & 14 & $0.009^{\mathrm{a}}$ \\
\hline Poor & 36 & 2 & & 35 & 3 & \\
\hline \multicolumn{7}{|c|}{ Lymph node metastasis } \\
\hline Yes & 38 & 2 & $0.000^{\mathrm{a}}$ & 34 & 2 & $0.005^{\mathrm{a}}$ \\
\hline No & 8 & 12 & & 9 & 15 & \\
\hline \multicolumn{7}{|l|}{ Liver metastasis } \\
\hline Yes & 15 & 0 & $0.014^{\mathrm{a}}$ & 13 & 0 & $0.010^{\mathrm{a}}$ \\
\hline No & 31 & 14 & & 30 & 17 & \\
\hline
\end{tabular}

${ }^{\mathrm{a}} \mathrm{P}<0.05$. HIF1- $\alpha$, hypoxia-inducible factor $1-\alpha$; TNM, tumor-node-metastasis.

Statistical analysis. Statistical analysis was performed using SPSS version 17.0 (SPSS, Inc., Chicago, IL, USA). Data are presented as the mean \pm standard deviation and Student's t-test and paired-samples t-test were performed as appropriate. The $\chi^{2}$ test or Fisher's exact test were used to evaluate any potential association between ARK5/HIF1- $\alpha$ expression and the clinicopathological parameters. Associations between expression of ARK5 and HIF1- $\alpha$ were analyzed using Spearman's correlation analysis. $\mathrm{P}<0.05$ was considered to indicate a statistically significant difference.

\section{Results}

Immunohistochemical staining of HIFl- $\alpha$ and ARK5, and correlation with clinicopathological features in human colon cancer. Immunohistochemical staining was used to determine the expression of HIF1- $\alpha$ and ARK5 in 60 patients with colon cancer, and HIF1- $\alpha$ and ARK5 were revealed to be overexpressed in colon cancer. Among these 60 colon cancer tissues, $76.7 \%$ (46/60) and 71.7\% (43/60) were positive for HIF1- $\alpha$ and ARK5 expression, respectively. Additionally, these two proteins were predominantly expressed in the cytoplasm and the nucleus (Fig. 1A). Single factor analysis demonstrated that expression of HIF1- $\alpha$ and ARK5 was associated with tumor stage (73.9 and $74.4 \%$, respectively), tumor grade (78.3 and $81.4 \%$, respectively), lymph node metastasis (82.6 and $79.1 \%$, respectively) and liver metastasis (32.6 and $30.2 \%$, respectively) (Table I). These results suggest that an association between HIF1- $\alpha$ and ARK5 may exist. Linear correlation analysis was then used for assessing the expression of HIF1- $\alpha$ and ARK5, and it was revealed that a correlation existed between the expression of these two proteins $(\mathrm{r}=0.776, \mathrm{P}=0.001)$ (Fig. 1B).

ARK5 is upregulated by HIF1- $\alpha$ under hypoxic conditions in the SW480 cell line. To study the association between ARK5 and HIF1- $\alpha$, the change in expression of ARK5 and HIF1- $\alpha$ was observed in the SW480 cell line under hypoxic conditions. During the period of hypoxia $(0,2,8$ and $24 \mathrm{~h})$, the mRNA level of ARK5 was markedly increased in the SW480 cell line, and reached its highest level at $24 \mathrm{~h}$ (Fig. 2A). Using western blot analysis to detect the change of protein expression in the SW480 cell line under the hypoxic conditions, the expression of HIF1- $\alpha$ was found to be increased and reached its highest level at $8 \mathrm{~h}$, while the expression of ARK5 was increased throughout the period of hypoxia (Fig. 2B). Such results demonstrate that ARK5 was upregulated under the hypoxic conditions in the SW480 cell line. To verify that ARK5 was regulated by HIF1- $\alpha$ under hypoxia, siRNA was used to suppress the expression of HIF1- $\alpha$ under the hypoxia condition, and both the mRNA and protein levels of ARK5 were markedly decreased (Fig. 2C and D).

HIF1- $\alpha$ promotes tumor proliferation and migration under hypoxic conditions by upregulating expression of ARK5. As ARK5 was upregulated by HIF1- $\alpha$ under hypoxic conditions and as HIF1- $\alpha$ may promote tumor proliferation and migration, it was suggested that there may be an association between ARK5 and tumor proliferation and migration under hypoxic 
$\mathbf{A}$

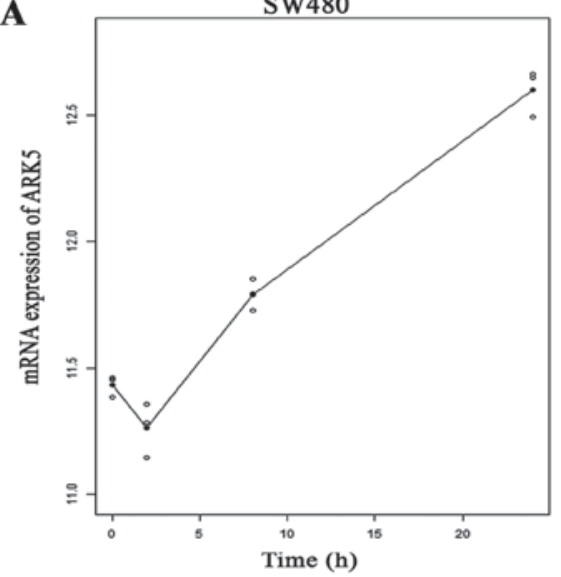

C

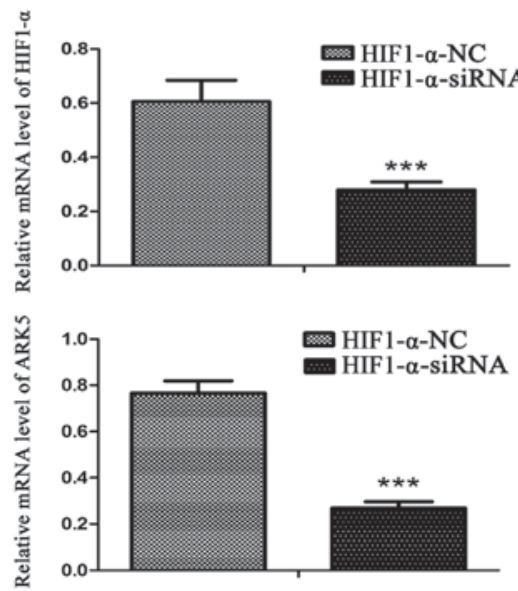

B
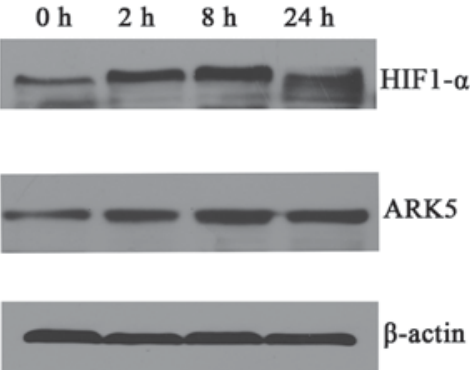

D
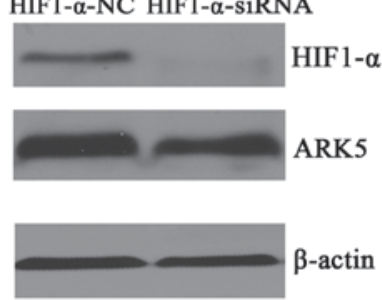

Figure 2. (A) Change in the level of ARK5 mRNA under hypoxic conditions in the SW480 cell line at 0,2,8 and $24 \mathrm{~h}$; (B) protein level of ARK5 and HIF1- $\alpha$ under hypoxic stress at $0,2,8$ and $24 \mathrm{~h}$; and ARK5 and HIF1- $\alpha$ (C) mRNA and (D) protein expression under hypoxic conditions. The SW480 cell line was treated with HIF1- $\alpha$ siRNA. ${ }^{* * *} \mathrm{P}<0.001$. HIF1- $\alpha$, hypoxia-inducible factor 1- $\alpha$. ARK5, AMPK-related protein kinase 5; siRNA, small interfering RNA; NC, negative control.

A

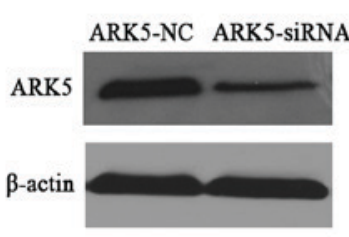

SW480 cell proliferation rate and apoptosis rate under hypoxic condition $\left(1 \% \mathrm{O}_{2}\right)$
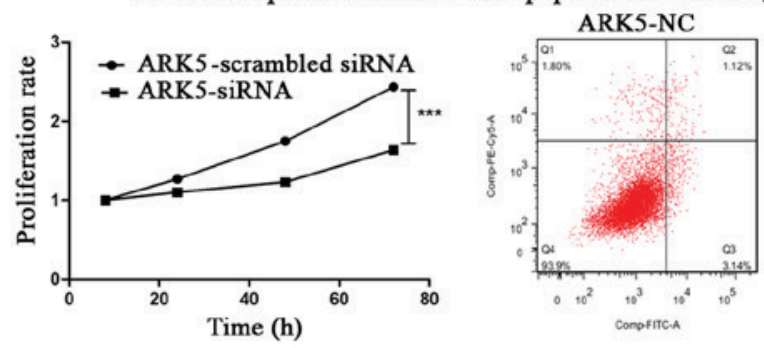
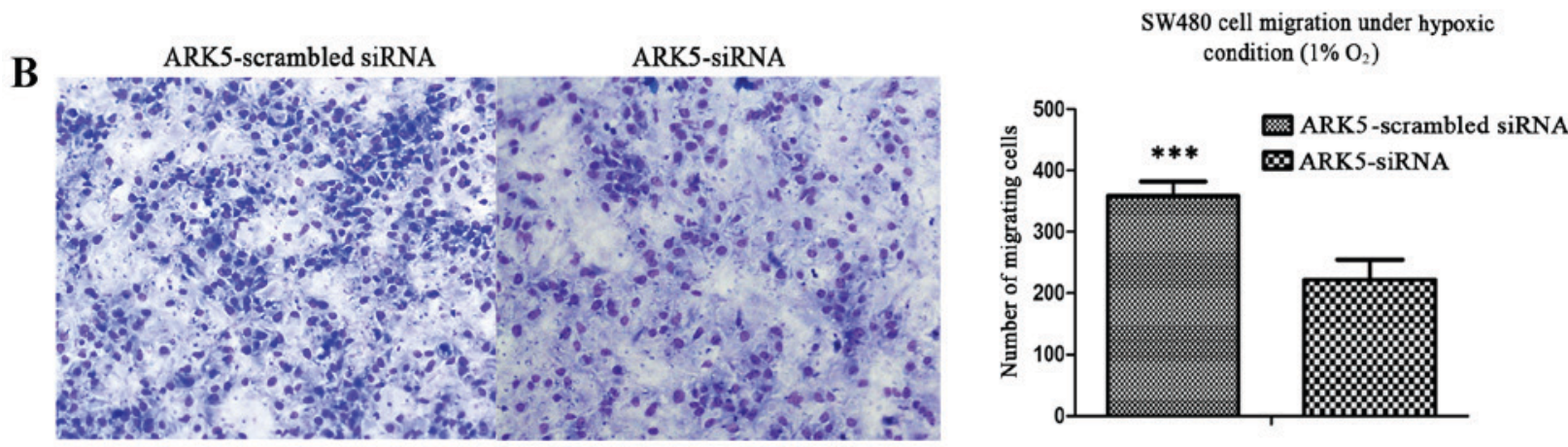

Figure 3. (A) Western blot analysis was used to detect the efficiency of ARK5 siRNA with scramble siRNA serving as a control. The viability of SW480 cells under hypoxic conditions for $8,24,48$ and $72 \mathrm{~h}$ was detected using a Cell Counting kit-8 assay and apoptosis was measured under hypoxic conditions at $48 \mathrm{~h}$. (B) The migration ability of the SW480 cells was detected under the aforementioned conditions. ${ }^{* * *} \mathrm{P}<0.001$. ARK5, AMPK-related protein kinase 5; siRNA, small interfering RNA; NC, negative control; FITC, fluorescein isothiocyanate; PE, phycoerythrin. 
conditions. To verify this hypothesis, siRNA was used to suppress the expression of ARK5, and cell proliferation, apoptosis and migration were observed by CCK- 8 , flow cytometry and Transwell assays under hypoxic conditions. These results demonstrated that the proliferation and migration abilities of the SW480 cell line were significantly decreased under the hypoxic conditions, while apoptosis did not differ (Fig. 3A and B). Therefore, ARK5 was the gene downstream of HIF1- $\alpha$ for proliferation and migration of the SW480 cell line under hypoxic conditions.

\section{Discussion}

Previous studies have suggested that ARK5 serves an important role in cancer proliferation, migration, invasion, starvation and drug resistance in a number of cancer types, including breast cancer, hepatocellular carcinoma, colorectal cancer and glioma, with ARK5 overexpression being associated with a poor prognosis $(5,9-12,21)$. However, the expression of ARK5 was not static but dynamic in response to conditions such as hypoxic stress. The role served by ARK5 in hypoxia remains unclear, as does the association between the expression of ARK5 and that of HIF1- $\alpha$.

As Kusakai et al (22) had demonstrated that ARK5 overexpression is involved in the progression of colon cancer, the present study only assessed the expression of ARK 5 and HIF1- $\alpha$ in cancer tissues. Based upon this, the present study demonstrated that ARK5 and HIF1- $\alpha$ were overexpressed in colon cancer, with linear correlations being identified. Furthermore, ARK5 was upregulated under hypoxic conditions over time in the SW480 cell line. Such findings suggest that ARK5 may be regulated by HIF1- $\alpha$ and may promote tumor cell survival during hypoxic conditions. To verify this hypothesis, we used siRNA to knockdown the expression of HIF1- $\alpha$ in the SW480 cell line under conditions of hypoxia. Notably, the expression level of ARK5 was also markedly decreased. These results demonstrated that ARK5 was upregulated by HIF1- $\alpha$ under conditions of hypoxia.

Hypoxia is a condition existing in all types of tumor and is involved in cancer proliferation, drug resistance, migration, invasion and immune escape. HIF1- $\alpha$ is serving a critical regulatory role under conditions of hypoxia (23-25). Analysis of the clinical data for 60 colon cancer patients revealed that the expression of ARK5 and HIF1- $\alpha$ was associated with tumor stage, tumor grade, lymph node metastasis and liver metastasis, and that ARK5 was downstream of HIF1- $\alpha$ under hypoxic conditions. We hypothesized that a partial function of HIF1- $\alpha$ under hypoxic conditions was conducted by ARK5 in colon cancer cells. SiRNA was used to knockdown expression of ARK5 in the SW480 cell line and, when the SW480 cell line was placed under hypoxic conditions, the proliferation and migration ability of the cells were significantly decreased compared with that of the negative control and such proliferation changes were not a result of apoptosis. Therefore, it can be concluded that HIF1- $\alpha$ promotes cell proliferation and migration under hypoxia in the SW480 cell line by upregulating expression of ARK5.

However, as the survival data of the aforementioned 60 patients was not available, it was not possible to analyze the tumor-free survival and overall survival associated with the co-expression of ARK5 and HIF1- $\alpha$. Therefore, further studies are required to address this issue. Additionally, the neighboring tissue was not analyzed as a control, as Kusakai et al (22) had previously demonstrated that ARK5 was overexpressed in colon tissue. To conclude, to the best of our knowledge, the present study was the first to identify an association between ARK5 and HIF1- $\alpha$ in colon cancer tissues, which may provide novel concepts for the clinical treatment of colon cancer.

\section{References}

1. WILD BWSCP and World Cancer Report: International Agency for Research on Cancer: 978-992-832-0443-5, 2014.

2. Siegel RL, Miller KD and Jemal A: Cancer statistics, 2016. CA Cancer J Clin 66: 7-30, 2016.

3. Board., P.A.T.E., PDQ Adult Treatment Editorial Board: Colon Cancer Treatment $\left(\mathrm{PDQ}^{\circledR}\right)$ : Patient Version. PDQ Cancer Information Summaries [Internet]. Bethesda (MD): National Cancer Institute (US), 2016.

4. Suzuki A, Lu J, Kusakai G, Kishimoto A, Ogura T and Esumi H: ARK5 is a tumor invasion-associated factor downstream of Akt signaling. Mol Cell Biol 24: 3526-3535, 2004.

5. Suzuki A, Kusakai G, Kishimoto A, Lu J, Ogura T and Esumi H: ARK5 suppresses the cell death induced by nutrient starvation and death receptors via inhibition of caspase 8 activation, but not by chemotherapeutic agents or UV irradiation. Oncogene 22: 6177-6182, 2003.

6. Suzuki A, Kusakai G, Kishimoto A, Shimojo Y, Miyamoto S, Ogura T, Ochiai A and Esumi H: Regulation of caspase- 6 and FLIP by the AMPK family member ARK5. Oncogene 23: 7067-7075, 2004.

7. Suzuki A, Kusakai G, Shimojo Y, Chen J, Ogura T, Kobayashi $\mathrm{M}$ and Esumi $\mathrm{H}$ : Involvement of transforming growth factor-beta 1 signaling in hypoxia-induced tolerance to glucose starvation. J Biol Chem 280: 31557-31563, 2005.

8. Suzuki A, Ogura T and Esumi H: NDR2 acts as the upstream kinase of ARK5 during insulin-like growth factor-1 signaling. J Biol Chem 281: 13915-13921, 2006.

9. Chang XZ, Yu J, Liu HY, Dong RH and Cao XC: ARK5 is associated with the invasive and metastatic potential of human breast cancer cells. J Cancer Res Clin Oncol 138: 247-254, 2012.

10. Cui J, Yu Y, Lu GF, Liu C, Liu X, Xu YX and Zheng PY: Overexpression of ARK5 is associated with poor prognosis in hepatocellular carcinoma. Tumor Biol 34: 1913-1918, 2013.

11. Sun X, Gao L, Chien HY, Li WC and Zhao J: The regulation and function of the NUAK family. J Mol Endocrinol 51: R15-R22, 2013.

12. Lu S, Niu N, Guo H, Tang J, Guo W, Liu Z, Shi L, Sun T, Zhou F, Li H, et al: ARK5 promotes glioma cell invasion and its elevated expression is correlated with poor clinical outcome. Eur J Cancer 49: 752-763, 2013.

13. Suzuki A, Iida S, Kato-Uranishi M, Tajima E, Zhan F, Hanamura I, Huang Y, Ogura T, Takahashi S, Ueda R, et al: ARK 5 is transcriptionally regulated by the Large-MAF family and mediates IGF-1-induced cell invasion in multiple myeloma: ARK5 as a new molecular determinant of malignant multiple myeloma. Oncogene 24: 6936-6944, 2005.

14. Semenza GL: Targeting HIF-1 for cancer therapy. Nat Rev Cancer 3: 721-732, 2003.

15. Wang GL, Jiang BH, Rue EA and Semenza GL: Hypoxia-inducible factor 1 is a basic-helix-loop-helix-PAS heterodimer regulated by cellular O2 tension. Proc Natl Acad Sci USA 92: 5510-5514, 1995.

16. Semenza GL: Defining the role of hypoxia-inducible factor 1 in cancer biology and therapeutics. Oncogene 29: 625-634, 2010.

17. Wang JS, Jing CQ, Shan KS, Chen YZ, Guo XB, Cao ZX, Mu LJ, Peng LP, Zhou ML and Li LP: Semaphorin 4D and hypoxia-inducible factor-1 $\alpha$ overexpression is related to prognosis in colorectal carcinoma. World J Gastroenterol 21: 2191-2198, 2015.

18. Amin MB, Greene FL, Edge S, et al: AJCC cancer staging manual.8th ed. New York: Springer: 252-274, 2017.

19. Livak KJ and Schmittgen TD: Analysis of relative gene expression data using real-time quantitative PCR and the 2(-Delta Delta C(T)) method. Methods 25: 402-408, 2001. 
20. Kong D, Su G, Zha L, Zhang H, Xiang J, Xu W, Tang Y and Wang Z: Coexpression of HMGA2 and Oct4 predicts an unfavorable prognosis in human gastric cancer. Med Oncol 31: $130,2014$.

21. Xu T, Zhang J, Chen W, Pan S, Zhi X, Wen L, Zhou Y, Chen BW, Qiu J, Zhang Y, et al: ARK5 promotes doxorubicin resistance in hepatocellular carcinoma via epithelial-mesenchymal transition. Cancer Lett 377: 140-148, 2016.

22. Kusakai G, Suzuki A, Ogura T, Miyamoto S, Ochiai A, Kaminishi M and Esumi H: ARK5 expression in colorectal cancer and its implications for tumor progression. Am J Pathol 164: 987-995, 2004.

23. Shin DH, Choi YJ and Park JW: SIRT1 and AMPK mediate hypoxia-induced resistance of non-small cell lung cancers to cisplatin and doxorubicin. Cancer Res 74: 298-308, 2014.
24. Mitani T, Ito Y, Harada N, Nakano Y, Inui H, Ashida H and Yamaji R: Resveratrol reduces the hypoxia-induced resistance to doxorubicin in breast cancer cells. J Nutr Sci Vitaminol (Tokyo) 60: 122-128, 2014.

25. Casazza A, Di Conza G, Wenes M, Finisguerra V, Deschoemaeker S and Mazzone M: Tumor stroma: A complexity dictated by the hypoxic tumor microenvironment. Oncogene 33: $1743-1754,2014$

(i) (9) This work is licensed under a Creative Commons Attribution-NonCommercial-NoDerivatives 4.0 International (CC BY-NC-ND 4.0) License. 